$11-1-2021$

Overcoming the challenges of community-engaged emergency referrals in a rural district of Pakistan

Imran Naeem Abbasi

Sameen Siddiqi

Follow this and additional works at: https://ecommons.aku.edu/pakistan_fhs_mc_chs_chs

Part of the Community Health and Preventive Medicine Commons, and the Health Services Research Commons 


\title{
Overcoming the challenges of community-engaged emergency referrals in a rural district of Pakistan
}

\author{
Imran Naeem Abbasi, Sameen Siddiqi
}

\begin{abstract}
A functional referral system for addressing health emergencies requires coordinated efforts by health system's stakeholders and the community. Using the Rural Health Programme (RHP) Thatta as a platform provided by an academic institution, district health system stakeholders and the community of Mirpur Sakro, taluka of Thatta, were engaged to strengthen the referral system for health emergencies through active community engagement. Through consultations with stakeholders, a referral system plan was developed with community volunteers from each village as focal persons for transportation, referring patients to health facilities and maintaining referral documentation. The stakeholders' experience and perceptions about the referral system were assessed through qualitative in-depth interviews. Patients' experiences of referral system improved with enhanced transport availability, but they faced structural challenges, including out of pocket transport expenses, weak backreferral links, lack of trust between the community and healthcare providers and poor availability of medicines.
\end{abstract}

Keywords: Implementation research, referrals, transportation, rural, Sindh, Pakistan.

\section{DOI: https://doi.org/10.47391/JPMA.011253}

\section{Introduction}

A functional referral system is an essential element of primary healthcare (PHC) that serves to connect the community with health facilities. Referral systems in many low- and middle-income countries, including Pakistan, face challenges 1,2 and the country faces major gaps in coverage and access to health services in rural areas, including a dysfunctional referral system.3,4 To address the challenges of the dysfunctional referral system, the Community Health Sciences Department, Aga Khan University (AKU) initiated the Rural Health Programme (RHP), an implementation science initiative aimed at health systems strengthening in the rural district of Thatta, Sindh. District health stakeholders and the community were brought together to collectively identify and address priority health issues. The lack of an effective referral system to address maternal

Community Health Sciences, Aga Khan University, Karachi, Pakistan.

Correspondence: Imran Naeem Abbasi. e-mail: imran.naeem2009@gmail.com and child health emergencies was highlighted as a key challenge. To address this challenge, health system stakeholders of the Thatta district were brought together to plan and implement a community engaged referral system.

Key stakeholders included the district health office (DHO) and the non-state providers (NSPs) working with the health department through public-private partnership (PPP) node and were managing public sector health facilities in the district. Through the mapping of the village and community-based organisations (CBOs), village representatives were approached to organise community meetings for building partnerships and apprise the communities regarding the importance of having a functional referral system. Private transporters of the respective villages were also mapped to aid in patient referrals.

\section{Developing and implementing a community-engaged referral system}

Consultation meeting of communities, NSPs, and DHO office was organised in October 2019. Stakeholders mentioned the weak role of community health workers, i.e., the lady health workers programme and lack of coordination among NSPs in rural areas as a key challenge and the need for the community's active participation in establishing a referral system. Lack of availability of transport was a major barrier. To address this, an NSP providing ambulance service under contract with PPP node and a mandate to provide ambulance services at doorsteps mainly for emergency cases was brought on board.

Referral system (figure) comprised of two community liaison persons in each of the 20 villages responsible for I) acting as a liaison between patient and the ambulance/private transporter; II) maintain documentation of referrals including providing a referral slip to the patients. A referral slip with details about the patient's name, age and the presenting complaint was designed and explained to the community liaison persons. A data recording form was also designed and handed over to the community liaison persons to maintain documentation of the referred patients. 


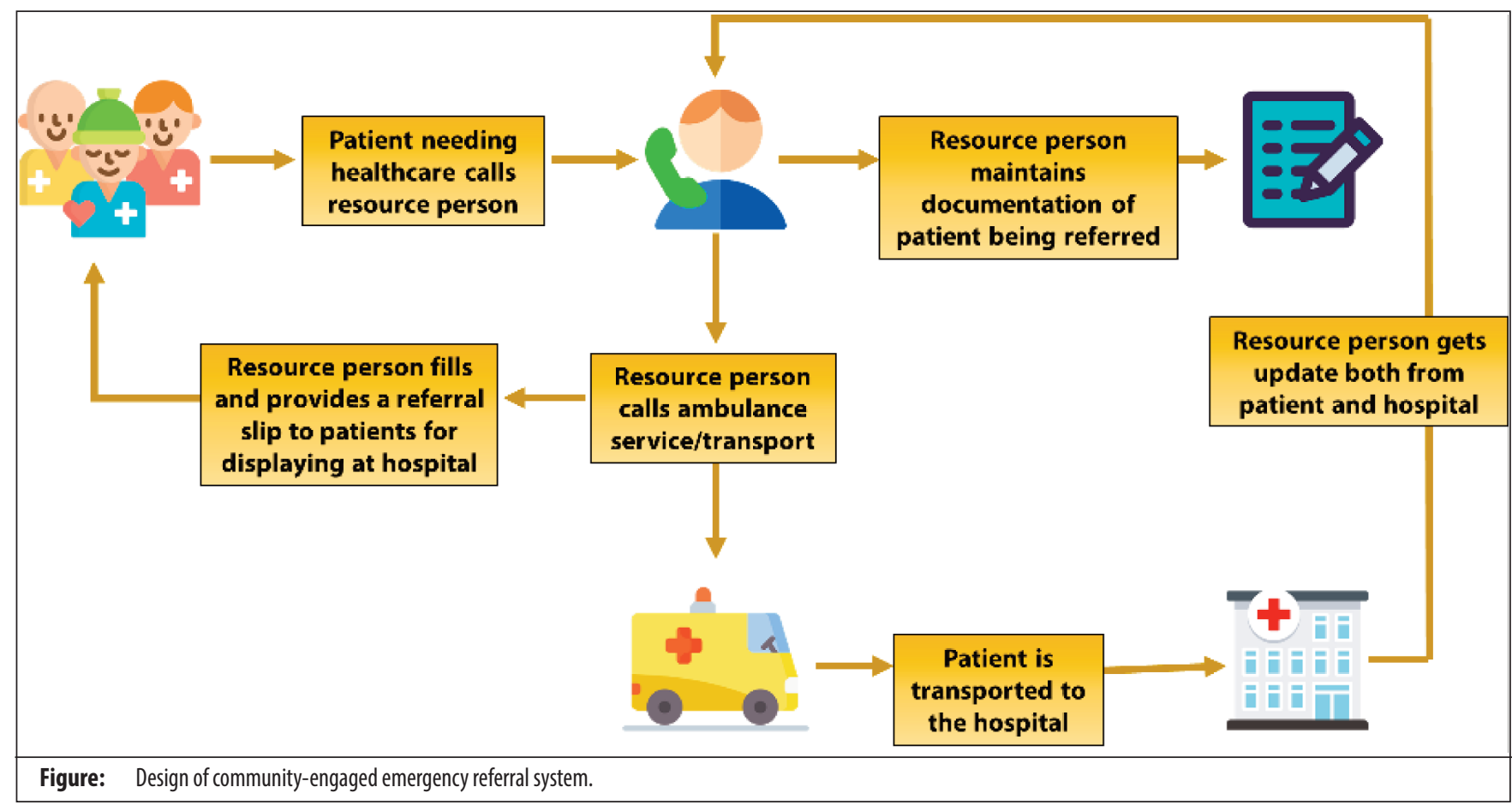

Following the successful implementation of the referral system, it was immensely important to delve into the minds of stakeholders, including the community and health service providers, to learn about their experience with the community-engaged referral system. Hence, the objective of this implementation research was to explore the stakeholders' experience and perceptions of the referral system.

\section{Methodology}

This study used a descriptive exploratory qualitative design to explore perceptions and experience of stakeholders with the community-engaged referral system six months following the implementation. Data was collected in February 2020.

Qualitative methods included 15 in-depth interviews (IDIs) with healthcare providers/managers and patients. Following a thorough literature search, a semi-structured IDI guide was developed to explore the perceptions and experiences of stakeholders regarding the referral system. Purposive sampling was used to select participants for indepth interviews. The interviews were conducted by an experienced researcher with a background in health systems research and qualitative research. The researcher moderated the interviews while a note-taker took notes.

Ethical considerations: Informed written and verbal consent was obtained from study respondents. Ethical approval for this study was obtained from the ethics review committee of the Aga Khan University, Karachi, Pakistan
(ERC \# 2019-0479-5202).

Data analysis: All the in-depth interview notes were translated from the local language (Sindhi) to English. Interview notes were read, and similar responses were merged and grouped into themes. The study themes were discussed among all the authors and discrepancies were resolved during the analysis and interpretation of the data.

\section{Results}

Referral system improved transport availability: Several cases were referred to health facilities through the referral system with the majority of these ending up at public sector hospitals with successful deliveries, treatment outcomes and/or referrals to the next level of care. All the patients termed the transport availability and facilitation by community liaison persons for transportation as a major improvement in their experience of referral system and health service utilisation. A patient described his experience as follows:

"I was very scared thinking about the usual lack of transport during late night hours and then getting to the hospital. But it all went very smoothly and timely." [mother - IDI]

Lack of trust was an important barrier in utilising free ambulance service: Despite the availability of free ambulance service in the city, the community was reluctant on a large scale in utilising the service for healthcare needs. This was evident from the fact that out of all the cases referred, only one patient used this service to reach the 
hospital. The general perception among the people of the area was that the service was created primarily for the privileged segment of the society only. One of the villagers stated the low response rate of ambulance service as the reason:

"We know that they won't serve us, hence, we do not call them and use private transportation to get to the hospital." [patient - IDI]

Sharing the patient experience during interviews, ambulance service providers said that the ambulance service was frequently misused by the community. The free ambulance service was primarily meant to serve emergency cases only and this had been shared with the community at large on several occasions. One of the respondents quoted:

"People call us even for mild illnesses such as fever, even though they are explained that the service is for emergency cases only. Given the limited resources, our motto is to cater to seriously ill patients on a priority basis." [healthcare provider - IDI]

Direct self-referrals to secondary hospitals were a constant ordeal: The community at large did not prefer going to primary healthcare facilities to address their healthcare needs. This was primarily influenced by the shorter working hours of primary healthcare facilities and the ready availability of some form of medication at secondary hospitals. One of the villagers responded:

"My younger son had a fever. We are poor and cannot afford to buy medication. I discussed it with the elders of my family and asked a friend and then decided to take my son to the big hospital." [father - IDI]

A mother-in-law shared her experience of her daughter-inlaw going through the labour pains as follows:

"It was past midnight and she started having pains. The nearest small public sector health facility was closed, and we had to rush to a big hospital to safely deliver the baby."

Referral links between facilities were weak: Almost all the respondents from the community said that they have been hardly ever advised to have a follow-up visit at a lower level (primary) healthcare facility while seeking treatment from secondary healthcare facilities. Only a few who sought care from secondary hospitals admitted to being provided with filled referral forms. A villager shared his experience:

"I asked the doctor, treating me for leg injury from a road accident if I can have my next visit at a small hospital near my house as it costs me transport to come to a big hospital. He just handed me over the prescription paper without further advice." [father - IDI]

Healthcare providers attributed the weaker referral links between primary and secondary healthcare facilities to a lack of coordination amongst stakeholders. A health manager responded:

"No mechanism has been set up to ensure back-referrals at primary healthcare facilities." [healthcare provider - IDI]

Structural health system challenges hindered referral system strengthening: Some patients were concerned about the lack of provision of medicines at the healthcare facilities. They thought that it would be futile to visit healthcare facilities if they do not get medicine from the hospital after seeing a doctor. A patient responded:

"The referral system, that you all are making efforts to establish, would not sustain if the patient reaches the hospital and is turned to pharmacies outside the hospitals only to spend out of pocket to buy medicines." [patient - IDI]

A healthcare provider said that sometimes patients have high expectations from public sector hospitals, and these are unrealistic. Public sector hospitals are under-resourced, and the patient load is very high.

\section{Discussion}

Our study shows that referral system was pivotal in improving the transport availability, however, lack of community's trust in the healthcare system, weaker links to ensure back-referrals between healthcare facilities and direct self-referrals to secondary hospitals and certain structural issues were key challenges experienced by community and healthcare managers alike while working with the community-engaged referral system.

Transport is an essential component of any referral system. Our study showed that respondents considered transport availability fundamental to the success of a referral system. These findings are similar to studies conducted in Mozambique, India, and Guatemala where strengthening transport service for referrals showed an increase in institutional deliveries. ${ }^{5-7}$

Despite the availability of free of cost ambulance service, most of the cases reached hospitals using private transportation. This led to out-of-pocket expenses on transportation. All respondents attributed it to a lack of trust in the service providers. Literature shows that publicprivate partnership to strengthen referral system has proven to be effective, ${ }^{8}$ however, trust remains an important determinant in choosing the type of service providers. ${ }^{9}$

Structural challenges such as limited working hours of 
primary healthcare facilities and reduced and/or nonavailability of medicines severely hampered the sustainability of the community-engaged referral system as experienced by the study participants. Studies from different countries have shown a similar impact of these structural challenges on continuity of care including the referral system. ${ }^{10}$

\section{Conclusion}

Involving both communities and stakeholders has the potential to strengthen the referral system. However, there is a need to concurrently address the structural challenges of the healthcare system.

Disclaimer: The article is part of a larger project titled: "Health Systems Strengthening for Improving Coverage and Quality: Capacity building, Health Surveillance and Targeted Interventions - Rural Health Programme Thatta". We have obtained the institutional IRB approval for this project and the same has been submitted to JPMA.

This is a sub-study from the larger project; hence the IRB approval is the same as granted for the larger project. The same is mentioned in the Methods section of the submitted article.

\section{Conflict of interest: None.}

Funding disclosure: This work was supported by a grant from World Health Organisation [Grant Number 51757].

\section{References}

1. World Health Organisation. Maternal mortality in 1990-2015. [Online] [Cited 2020 Oct 01]. Available from: URL: https://www.who.int/gho/maternal_health/countries/pak.pdf?ua=1.

2. Pasha O, Saleem S, Ali S, Goudar SS, Garces A, Esamai F, et al. Maternal and new-born outcomes in Pakistan compared to other low and middle income countries in the Global Network's Maternal New-born Health Registry: an active, community-based, pregnancy surveillance mechanism. Reprod Health. 2015; 12:S15.

3. Afsar HA, Qureshi AF, Younus M, Gulb A, Mahmood A. Factors affecting unsuccessful referral by the lady health workers in Karachi, Pakistan. J Pak Med Assoc. 2003; 53:521-8.

4. Afsar HA, Younus M, Gul A. Outcome of patient referral made by the lady health workers in Karachi, Pakistan. J Pak Med Assoc. 2005; 55:209-11.

5. Give C, Ndima S, Steege R, Ormel H, McCollum R, Theobald S, et al. Strengthening referral systems in community health programs: a qualitative study in two rural districts of Maputo Province, Mozambique. BMC Health Serv Res. 2019; 19:263.

6. Prinja S, Jeet G, Kaur M, Aggarwal AK, Manchanda N, Kumar R. Impact of referral transport system on institutional deliveries in Haryana, India. Indian J Med Res. 2014; 139:883-91.

7. Kapoor R, Avendaño L, Sandoval MA, Cruz AT, Sampayo EM, Soto MA, et al. Initiating a standardised regional referral and counter-referral system in Guatemala: a mixed-methods study. Glob Paediatr Health. 2017; 4:2333794X17719205.

8. Joloba M, Mwangi C, Alexander H, Nadunga D, Bwanga F, Modi N, et al. Strengthening the tuberculosis specimen referral network in Uganda: the role of public-private partnerships. J Infect Dis. 2016; 213:S41-6.

9. Amoah PA, Phillips DR. Strengthening the referral system through social capital: a qualitative inquiry in Ghana. Healthcare (Basel). 2017; 5:80.

10. Patel S, Awoonor-Williams JK, Asuru R, Boyer CB, Tiah JA, Sheff MC, et al. Benefits and limitations of a community-engaged emergency referral system in a remote, impoverished setting of northern Ghana. Glob Health Sci Pract. 2016; 4:552-67. 